\title{
Reflets
}

Revue ontaroise d'intervention sociale et communautaire

\section{L'État de la situation des services de santé mentale pour enfants en Ontario - Entrevue avec Michelle Bernier-Wilson}

Volume 7, numéro 1, printemps 2001

Santé mentale et les défis de l’an 2001

URI : https://id.erudit.org/iderudit/026334ar

DOI : https://doi.org/10.7202/026334ar

Aller au sommaire du numéro

Éditeur(s)

Reflets : Revue ontaroise d'intervention sociale et communautaire

ISSN

1203-4576 (imprimé)

1712-8498 (numérique)

Découvrir la revue

Citer cet article

(2001). L'État de la situation des services de santé mentale pour enfants en

Ontario - Entrevue avec Michelle Bernier-Wilson. Reflets, 7(1), 18-23.

https://doi.org/10.7202/026334ar

Tous droits réservés (C) Reflets : Revue ontaroise d'intervention sociale et communautaire, 2001
Ce document est protégé par la loi sur le droit d'auteur. L'utilisation des services d'Érudit (y compris la reproduction) est assujettie à sa politique d'utilisation que vous pouvez consulter en ligne.

https://apropos.erudit.org/fr/usagers/politique-dutilisation/ 


\section{L'État de la situation des services de santé mentale pour enfants en Ontario}

Cette entrevue a été réalisée auprès de Michelle Bernier-Wilson, coordonnatrice des programmes pour les services francophones au Centre de l'enfant et de la famille de Sudbury depuis dix ans.

Michelle a plus de vingt années d'expérience dans le domaine de la santé mentale. En plus de son travail au Centre de l'enfant et de la famille, elle est aussi en pratique privée. Elle s'intéresse principalement aux personnes ayant subi un traumatisme. Elle fait également partie de l'équipe responsable du processus d'agrément pour l'Association de la santé mentale pour enfants en Ontario. Michelle détient une Maîtrise en service social de l'Université Laurentienne.

Reflets : Pouvez-vous nous tracer un bilan général de la situation actuelle en Ontario sur les services de santé mentale pour enfants?

Michelle : Il y a 90 centres de santé mentale pour enfants en Ontario, qui servent 150000 enfants et adolescents et leurs familles à chaque année. Par contre, à travers la province, plus de 8000 enfants avec des besoins critiques demeurent sur des listes d'attente. Nous trouvons ces enfants dans toutes les communautés et à tous les niveaux économiques de la province.

Au niveau financier, depuis 1993, l'investissement du gouvernement dans le financement de base des services de santé mentale a été réduit de $8 \%$. La hausse dans le coût de vie ainsi que l'équité salariale non financée par le gouvernement ont fait que les Centres ont dû faire face à une réduction de 20 à $25 \%$ en vrais dollars depuis 1990. Le recrutement et la capacité de garder 
le personnel qualifié est un des plus grands défis. Les salaires dans les organismes communautaires des centres de santé mentale pour enfants sont à peu près $30 \%$ plus bas que les salaires dans les hôpitaux et les services gouvernementaux. L'érosion du personnel avec de l'expérience produit des environnements de travail où il y a moins de créativité et d'innovation et des situations où le personnel moins expérimenté soigne les enfants les plus perturbés. Avec moins d'after-care et de suivi, les progrès réalisés durant le traitement ou les placements résidentiels sont perdus.

En même temps, les centres ont réussi à garder leurs coûts administratifs en dedans des limites du $10 \%$. Ces réductions furent produites en augmentant notre efficience, en utilisant des employés à temps partiel et en cherchant des sources alternatives de financement.

Reflets: Quels sont les dossiers d'importance actuellement dans la province au sujet de la santé mentale des enfants?

Michelle: En l'an 2000, le gouvernement a fait un investissement de 20 \$ millions pour des services de santé mentale pour enfants. Cet argent a été dirigé vers des initiatives particulières. Il y a, d'abord, les Services mobiles d'intervention d'urgence, qui est un programme d'intervention de crise, 24 heures sur 24, sept jours par semaine. Ce programme aide les enfants, les adolescents et leurs familles à accéder aux services nécessaires immédiats (consultation et avis téléphonique, services d'intervention qui se rendent à la scène de la crise, placement à court terme dans une résidence).

Il y a aussi les services intenses. Ce sont des services à plus long terme, réalisés en collaboration avec les écoles et les hôpitaux, qui ciblent les enfants et les adolescent à risque élevé, témoins de violence et qui sont isolés et incapables de fonctionner dans le domaine scolaire.

La télépsychiatrie est un autre programme qui utilise la technologie pour fournir de la consultation et de la formation aux professionnels de santé mentale, ciblant particulièrement les régions rurales de l'Ontario. 
Finalement, il y a le système d'information. Ce système permet de faire l'administration à travers tous les centres de la province, à l'aide d'instruments standardisés, pour documenter la nature et la sévérité des problèmes en Ontario et pour mesurer les résultats de nos services.

Reflets : Est-ce que le gouvernement est engagé ou désengagé vis-à-vis les services de santé mentale aux enfants? Quelles sont les implications de ceci?

Michelle : La vision du ministère des Services sociaux et communautaires est plus large et englobe les besoins des enfants en général. Le réseau des Centres de santé mentale pour enfants n'est qu'un petit élément de tous les services dédiés à l'enfance. Je crois qu'il y a une appréciation que les déterminants de la santé mentale se situent aussi dans les domaines de la santé et de l'éducation, et c'est en partie pourquoi le ministère des Services sociaux et communautaires, en partenariat avec le ministère de la Santé et des soins de longue durée et le ministère de l'Éducation, a créé une division nouvelle des Services intégrés à l'enfance, laquelle assure que le développement de politiques et la planification de programmes soient intégrés et coordonnés. Il faut dire que le gouvernement a récemment parrainé plusieurs initiatives qui ont généré des résultats positifs pour les jeunes enfants. À la lumière de l'évidence des impacts de la recherche du Dr Fraser Mustard, les initiatives de «Bébé en santé» et d'orthophonie pour les jeunes d'âge préscolaire ont été bien reçues par les organismes communautaires.

Localement, le ministère des Services sociaux et communautaires est très impliqué dans la mise en place du modèle MSWP (Making Services Work for People - Pour des services au service des gens) et aussi au niveau du développement de la Co-op. Cette Co-op est un regroupement qui englobe plusieurs organismes afin d'établir une plus grande collaboration entre tous les partenaires dans le réseau des services aux enfants. C'est une initiative unique au nord ontarien.

Reflets: Quel est le mandat du Centre de l'enfant et de la famille?

Michelle : D'offrir des services de santé mentale aux enfants de moins de 18 ans et à leur famille, tout en respectant leurs besoins linguistiques 
et culturels. Nos services incluent des services de thérapie individuelle et familiale, en clinique externe, l'intervention de crise, les services de traitement de jour dans les écoles, des programmes résidentiels, un programme de thérapie de groupe pour parents et jeunes. Nous consultons régulièrement les écoles, les Sociétés de l'aide à l'enfance, les garderies, la police et d'autres organismes qui travaillent auprès des enfants, mais qui ne sont pas des experts en santé mentale de l'enfant.

Reflets: Qui sont les enfants que vous servez?

Michelle: Les enfants et les adolescents que nous servons souffrent de problèmes socio-affectifs ou de santé mentale. Les signes et les symptômes varient de la dépression, du comportement suicidaire, de l'agression, de la victime de traumatisme, que ce soit l'abus sexuel, physique, émotionnel, de témoins de violence familiale jusqu'à la psychose ou à la toxicomanie.

Reflets: Avez-vous les ressources nécessaires pour remplir votre mission adéquatement?

Michelle : Non.Au niveau provincial, plus de 8000 enfants avec des besoins critiques demeurent sur des listes d'attente.

Reflets: On a l'impression que la clientèle qui requiert des services comme les vôtres a beaucoup changé ces dernières années. Est-ce le cas et pouvezvous nous expliquer la différence?

Michelle: Depuis l'implantation de l'initiative Pour des services au service des gens, en 1997, nous nous devons de servir les populations les plus en besoin. Celles-ci sont les enfants et les adolescents exposés à un grave danger en raison d'un trouble affectif ou de comportement. Ce sont aussi les enfants et les adolescents qui se sont nui à eux-mêmes ou à autrui ou qui se sont exposés à un danger grave et imminent. Il y a les enfants et les adolescents dont la famille est en danger imminent d'éclatement sans la prestation de mécanismes de soutien.

Les familles qui accèdent à nos services sont à multi-problèmes et les enfants sont plus perturbés. Ils nécessitent des services plus intenses, plus ingérents, une plus grande variété de services et pendant une plus grande période de temps. 
Reflets: Par exemple, est-ce vrai qu'on sert des enfants de plus en plus jeunes?

Michelle: Les jeunes enfants qui sont éligibles aux services sont plus perturbés.

Reflets: Est-ce que les services s'étendent également aux familles?

Michelle: Le réseau des Centres de l'enfant et de la famille a toujours préconisé une approche systémique familiale et le travail avec et auprès des parents et des familles est central à sa vision et dans les méthodes de travailler pour les enfants.

Reflets : De quoi aurait-on besoin pour améliorer la livraison des services de façon plus efficace?

Michelle: Un bon nombre d'initiatives ont déjà été mises en place. Par contre, Santé mentale pour enfants Ontario, l'organisme provincial qui représente les centres de santé mentale pour enfants, fait demande pour une allocation additionnelle de $30 \$$ millions afin de développer et d'implanter des programmes pour rencontrer les besoins des bébés et des enfants d'âge préscolaire avec des problèmes de santé mentale. L'association provinciale fait aussi une autre demande de $30 \$$ millions pour travailler dans les milieux scolaires.

Reflets: Les services à la population francophone sont-ils comparables à la population anglophone? Quelles distinctions faites-vous aussi avec les services à la population autochtone?

Michelle : Comme minorité nous sommes sur-représentés quant aux facteurs de risques, aux taux de pauvreté plus élevés, aux taux de scolarité moins élevés, au nombre moins élevé de services collatéraux en français.

L'équipe autochtone est plus petite avec seulement trois cliniciens et une gérante à demi-temps. C'est une population qui est aussi sur-représentée, même plus que la population francophone. Plusieurs Autochtones arrivent des réserves et ils ont de la difficulté à s'acclimater à un centre urbain. De plus, dans l'ensemble, ils souffrent de problèmes médicaux plus sévères et le taux de pauvreté est très élevé. Par contre, la population autochtone possède un bon réseau avec le Centre de santé communautaire et le Friendship Centre. Comme groupe, il est aussi plus efficace dans 
l'utilisation des réseaux volontaires de la communauté, où l'approche traditionnelle autochtone est utilisée avec la participation des aînés.

Reflets: Comment peut-on sensibiliser le grand public à la cause des services de santé mentale pour enfants en Ontario?

Michelle: Bonne question. Souvent les symptômes des problèmes de comportement (l'agression verbale et physique, la violence, la délinquance, la toxicomanie) font en sorte que c'est une population à vendre, «faire du marketing». C'est une population que les gens préferent ne pas voir. Dans les écoles, avec la nouvelle législation qui permettra aux enseignants de suspendre les jeunes agressifs, on est plus vite à éjecter le problème qu'à y mettre des ressources pour résoudre le problème. 